\title{
Study on the Contribution of Xiling's Poetry to Poetry Flourished in Earlier Qing Dynasty
}

\author{
Liping $\mathrm{Gu}$ \\ The Engineering \& Technical College of Chengdu University of Technology, Leshan, Sichuan, \\ 614000
}

Keywords: Xiling Poetry, Poetry Flourished, Qing Dynasty

\begin{abstract}
At the beginning of the Qing Dynasty, Xiling language refers to the late Ming and early Qing Dynasty in Xiling a word activities of the word group, including Xiling capital of the poet, including the official travel in Xiling's poetry, is a geographical, The family, the teacher as a link to the end of the alliance as an opportunity, while infiltration Xiling heavy word tradition, in the late Ming Dynasty poetry specific poetry language formation in the group of people. They are more rational and objective theory, especially emphasizing the essence of the word speculation, pay attention to the word rhyme and the creation of the law of the summary, whether it is theory or creation, the development of the Qing Dynasty have far-reaching impact. In short, the late Ming and early Qing Dynasty Xiling language in the word from the yuan, the decline since the turn of the Qing Dynasty to the revival of the evolution of this process is a can not be ignored.
\end{abstract}

\section{Introduction}

The formation and development of the Xiling School are inseparable from the development of the same language. Many people regard the Western Cold School as the rise of the cloud and even as part of the cloud. Such as "Qing Dynasty" records: "first Chen Zilong for Denglou community, folding, and in the same Chai Shao Bing, Mao Xianshu, Sun Zhi, Zhang Dan, Wu Bai Peng, Shen Qian, Yu Huang Hao and so on, the name. Zhu Qinghu said:" Mr. Chen Laozai Secretary Li Shaoxing, poems are Sheng, Zhejiang East and West who are not in accordance with its instructions, so Zhang Gang Sun and other writers written by Xiling Shi Zi, Jun Yun asked also. " Said: "Xiling Jiyun between." Wu Weiying said: "cloud between the philosophers following the food state and name also, Long sleep Xiling following the cloud asked the author also."

"Ten Xiling Wits" is the late Ming and early Qing Dynasty in Hangzhou, the greater influence of the poetic groups, ten poets are: Mao Xianshu, Lu Qi, Chai Shao Bing, Ding Peng, Shen Qian, Sun Zhaobu, Wu Baipeng, Chen Tinghui, Zhang Dan, Yu Huanghao. Xiling also wrote Xiling, the ancient name of Hangzhou, while the West Lake also has a bridge called Xiling Qiao. Initially, under the influence of Chen Zilong, Lu Qi and others formed a poetry club on the lake, after Mao Xianshu and Chai Shao Bing jointly compiled "Ten Xiling Wits", "Ten Xiling Wits" in the name of the spread to go. Their poems are far from before and after the seven children, close to catch the clouds between the school of poetry to Tang poetry as the object. "Ten Xiling Wits" members of the rich, only "Ten Xiling Wits" to close nearly 900 poems, in addition, ten son and other works handed down, a larger number of poems. However, few of these works touched, in addition, there are many on the "Ten Xiling Wits" information to be explored. At present, for the "Xiling Xizi" research has accumulated a lot of results, on this basis, how can from the perspective of literati and "Ten Xiling Wits" for a more comprehensive criticism, deepen the "Ten Xiling Wits" The formation of the poem and its influence and so on, will be able to more fully understand the "Ten Xiling Wits" of the formation process and its poetic view of the specific content, and for the understanding of its poetry The impact is also helpful. To a certain extent, will enrich the existing research results, and later for researchers to learn from. This is the basis of this topic and the meaning lies.

\section{The Communication Characteristics of Ten Xiling's Wits}

"Ten Xiling Wits," the majority of members from the Ming and Qing dynasties, seclusion writings, 
communication activities are mainly confined to the region of Jiangsu and Zhejiang, so its range of contacts is not large. However, due to the background in the late Ming and early Qing Dynasty, "Ten Xiling Wits" across two dynasties, the communication characteristics of the times to bring some of the times. "Ten Xiling Wits" communication characteristics can be investigated from three aspects. "Xiling Xizi" is not a coincidence, is based on a common geographical environment, similar to the creative ideas and aesthetic pursuit, the family origins and many other factors. "Ten Xiling Wits" internal members can be described as "also friends, also teacher, also pro." "Ten Xiling Wits" members because of friendly, and par. Mao Xianshu, Shen Qian, Zhang Dan Zeng in the south of the house in the south of the Lou Xiao Gao Yin, wine wine wind, pay homage to the ages, when people called "South House three sons", Lu Qi also participated in the three men singing and. Wu Bai Peng Ren Nan and county magistrate, died in the official, Sun Zhi personally for their funeral, and tending their children adult. The friendship between the two is self-evident. Ding Peng has been under the tutelage of Wu Bai Peng, Lu Qi for Shen Qian teacher, internal members of the division of close relationship. In addition, Sun Qi and Lu Qi have in-laws, Sun Zhitang for Lu Qi's wife. Lu Qi and Wu Bai Peng for the family, Lu Qi's second daughter, married in the eldest son of Wu Baipeng. After the death, Lu Qi's brother Lu Pei martyred, Chen Ting will be carefully taught, tending the land of Lu Pei Lu. Mao Xianshu and Zhang Dan's brother Zhang Zuding relationship is also quite close, two often have poetry exchanges. "Ten Xiling Wits" members of the relationship beyond the general friends, and thus more intimate, this intimate relationship will contribute to the transmission of literary activities, but also bring some drawbacks, to a certain extent, will lead to poetic theory of succession, and thus Lack of vitality of innovation.

\section{The Characteristics of the Poetic Creation of Ten Xiling Wits}

For the vast number of scholars, the Ming Dynasty, China is undoubtedly a huge catastrophe. Subjugation of sorrow filled their hearts, a long time can not be discharged and relieved. This period of literati, or climb overlooking, or line Yin Ze, or visit the ancient visit, or poetry wine wind, in their own way to express the sadness of the heart, to express the pain of subjugation, the country of thinking. "Ten Xiling Wits" is also true. Although the "Ten Xiling Wits" is not strictly in the sense of the group of people, but the comprehensive "Adherent Poetry", "Ming Min recorded", "Ming Shi Chronicle" and "Qing Shi Chronicle" , May be Mao Xianshu, Lu Qi, Chai Shao Bing, Shen Qian, Sun Zhi, Chen Tinghui, Zhang Dan seven people as the list of the survivors, at the same time, later the new DPRK Ding Peng, Wu Bai Peng, Yu Huanghao three people and the descendants of the contact is also thousands of silk Wanlie. On the occasion of the occasion, the soldiers and raindrops, they have personally experienced this catastrophe, therefore, the poetry of his country's thinking and the pain of subjugation will be self-evident.

Ming and Qing dynasties caused by not only the poet's inner sadness and sorrow, the poet in the sadness, but also to look into a more profound society, concerned about the suffering of the people of the people. In the case of frequent war, the poet's inner anguish and sorrow often need friendship comfort, "Ten Xiling Wits" is also no lack of cross-singing and singing, singing and singing is to maintain friendship ties. "Ten Xiling Wits" relationship is more closely, frequent interaction within the members of the interaction. Sun Jiu in Wu Bai Peng died after the South, for their cooking funeral, and take care of their children grow up. And Sun Zhi personally taught Lu Qi from the sub-Lu Fan, training Lu Fan literary accomplishment. Among them, Mao Xianshu, Zhang Dan, Shen Qian also claiming to be "South House three sons", or "Qiantang three sons", three people often set in the Shenqian house in the south floor, all night, poetry wine talk, enjoy and go. For each poet's poetic genre analysis, Mao's first six poems accounted for more than $90 \%$ of the total number of poems. According to the above table, the proportion of the above nine poets in the total number of poems $\%, 85 \%, 80 \%, 72 \%, 72 \%, 40 \%, 96 \%, 91 \%, 98 \%$. It is not difficult to see that only Lu Qi and Wu Baipeng the number of the six categories of poetry accounted for the proportion of the total number of poetry is small, the remaining eight are more than 70 percent. 


\section{The Impact of Ten Xiling Wits}

"Ten Xiling Wits" and the cloud between the leaders of Chen Zilong close, "Ten Xiling Wits" is under the direct influence of Chen Zilong appeared. On the relationship between "Ten Xiling Wits" and the cloud to send Chen Zilong, Yang Zhongxi in the "snow bridge poetry first episode" volume a clear that: "Chen lying son of Li Shaoxing, poems both Sheng, Zhejiang East and West are all non-compliance In addition, with the "Ten Xiling Wits" close poet Wu Weiye in the "Fu Fu community book" Yiyun: "Xiling, following the cloud between the clouds," Xiling, And the author also. "Here," Xiling "on behalf of" Ten Xiling Wits ". "Ten Xiling Wits" and the cloud to send Chen Zilong close, and "Ten Xiling Wits" poetic concept inherited Chen Zilong's poetic view, is an indisputable fact, but if so will "Ten Xiling Wits" Into the cloud branch of the branch, will largely cover up the "Ten Xiling Wits" and his poetic view at the time of the status and influence of poetry.

In fact, in the Qing Dynasty poetry, "Ten Xiling Wits" status and influence quite. Zhang Qianyi in the "Zhai Zhai talk" said: "Zhang Xiaoyi will" Ten Xiling Wits " And the Ming Dynasty after the seven sub, Jing Ling faction, cloud sent between par, see "Ten Xiling Wits" status of the heavy. In addition, Wu Weiyong in the "Taicang ten poem preface," said: "This is the son of the sub-Zi, since the sub-Chu, are all with the clouds, Xiling Zhuzi up and down its can." Wu Weiying this discourse, intended to emphasize Taicang ten sub-status of the importance of his side from the side of the "Ten Xiling Wits" and the cloud sent a close relationship at the same time, but also affirmed the "Ten Xiling Wits" status of the importance.

As a regional poetry genre, "Ten Xiling Wits" of the main activities concentrated in the region of Jiangsu and Zhejiang, in the two Zhejiang poetry, "Ten Xiling Wits" to the leader of the attitude of active in the poetry for decades. In the late Ming to the early Qing Dynasty in the process of historical changes, the two Zhejiang poetry also will change. "Xiling Xizi" in this process, in the bearing on the status of criticism in the succession of predecessors on the basis of the theory, adhere to their own retro Chong Tang advocated, affecting the creative atmosphere of Hangzhou poetry, such as Mao Qiling said, in the " Ten Xiling Wits "active in the two Zhejiang poetry period, poet scholar" did not dare to Song Yuan's poem into the Xiling sector ", is" Ten Xiling Wits "credit.

First of all, "Ten Xiling Wits" influence banana garden poetry society. Qing Dynasty Wang Yunzhang "Ran Yu Yun Yun" records: "Qiantang banana garden seven children, Lin Yining Qing, Gu Qi Ji Si, Chai Ji Xian Jing instrument, Feng You Ling Xian, Qian Yunyi Feng Lun, Zhang Chaoyun Hao, Mao Anfang is also. "Banana garden poetry by Chai Jingyi, Zhang Hao, Mao and other seven people formed, during the mutual singing and, Chai Jingyi for the poetry of the sponsor and host. Chai Jingyi, Mao, Zhang Hao and the three "Ten Xiling Wits" closely linked. First look Chai Jingyi. Chai Jingyi, word Ji Xian, Renhe people, Xiao Lian Chai Yunqian second woman, Chai Shao Bing niece, Shen Hanjia wife. Author of "condensate room poetry money" two volumes, "North Church set". Ding Peng has been ordered by the book: "My friend Shen Hanjia wife Chai quarter Xian, young and intelligent, workers for the poem, Mr. Fu Yunqian gentleman personally lessons, while the season is particularly clear, than Liu Xiaochao three sister that season also "According to Hu Wenkai" ancient works of women test ", Mao Xianshu, Ding Peng have been Chai Jingyi's" condensate room poetry money notes. "Order. "Ten Xiling Wits" on the Hangzhou poetry and the younger generation of the impact, such as Li E said, "the wind is not falling too." As a poetic group, from the late Ming Dynasty Chongzhen to the early Qing Dynasty, although experienced the pain of subjugation, but "Ten Xiling Wits" does not waste poetry creation, based on poetry society active in Hangzhou poetry, and friends poetry Wine advocacy, poetry works a large number of influential quite.

\section{Conclusion}

The group of Xiling's poetry people especially emphasizes the speculative nature of the vocabulary, emphasizes the summary of the rhyme and the creation of the law, whether it is theory or creation, has profound influence on the development of the Qing poetry, such as later And Zhexi poetry School poetry theory, can be found from the Xiling word group where the source. Therefore, the 
theory of poetry language of Xiling's poetry - poets has both the imprint of the thought of the Ming Dynasty and the new philosophical thought, which is the key part of the evolution of the Ming and Qing dynasties. It is of great significance to study the theory of poetry in the Ming and Qing Dynasties and the rejuvenation of the Qing dynasty.

\section{References}

[1] Sun Xutang. "Proposed" "change" and literary retro [J]. Book of Changes, 2011 (04)

[2] Jiang Yin. Qiao Tong poet and Mao Qiling's poetic tendency in the early Qing Dynasty [J]. Hunan Social Sciences, 2008 (05)

[3] Sun Keqiang, Yue Shuzhen. Mao Xianshu theory of shortness [J]. Journal of Nankai University, 2008 (04)

[4] Li Kanghua. Shen Qian, Mao Xianshu's similarities and differences in thought [J]. Chinese Journal of Yunwen, 2002 (01)

[5] Yang Changchun. Qing Dynasty poet Ding Peng's birth and death test [J]. Journal of Ningxia University, 1986 (03)

[6] Ding Shengjun. Early Qing Dynasty poet Ding Peng [J]. Nationala University Journal, 1980 (04) 